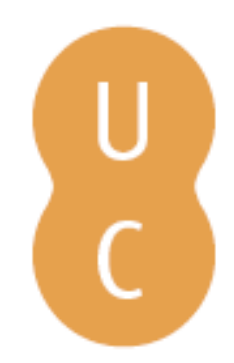

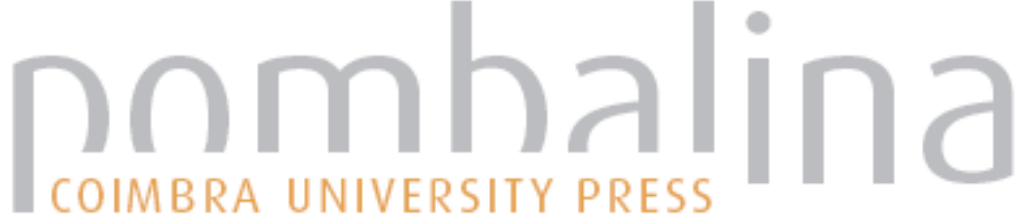

\section{La propaganda fascista in Europa: alcune riflessioni}

\author{
Autor(es): $\quad$ Bernardi, Alberto De
}

Publicado por: Imprensa da Universidade de Coimbra

URL

persistente: URI:http://hdl.handle.net/10316.2/38995

DOI: $\quad$ DOI:http://dx.doi.org/10.14195/978-989-26-1064-1_1

Accessed : $\quad$ 26-Apr-2023 15:45:11

A navegação consulta e descarregamento dos títulos inseridos nas Bibliotecas Digitais UC Digitalis, UC Pombalina e UC Impactum, pressupõem a aceitação plena e sem reservas dos Termos e Condições de Uso destas Bibliotecas Digitais, disponíveis em https://digitalis.uc.pt/pt-pt/termos.

Conforme exposto nos referidos Termos e Condições de Uso, o descarregamento de títulos de acesso restrito requer uma licença válida de autorização devendo o utilizador aceder ao(s) documento(s) a partir de um endereço de IP da instituição detentora da supramencionada licença.

Ao utilizador é apenas permitido o descarregamento para uso pessoal, pelo que o emprego do(s) título(s) descarregado(s) para outro fim, designadamente comercial, carece de autorização do respetivo autor ou editor da obra.

Na medida em que todas as obras da UC Digitalis se encontram protegidas pelo Código do Direito de Autor e Direitos Conexos e demais legislação aplicável, toda a cópia, parcial ou total, deste documento, nos casos em que é legalmente admitida, deverá conter ou fazer-se acompanhar por este aviso.

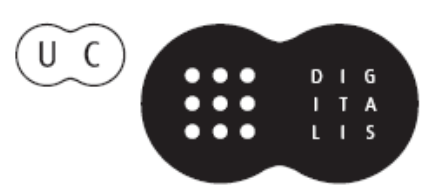


A L B ERTO PENA-RODR ÍGUEZ HELOISA PAULO

COOR D.

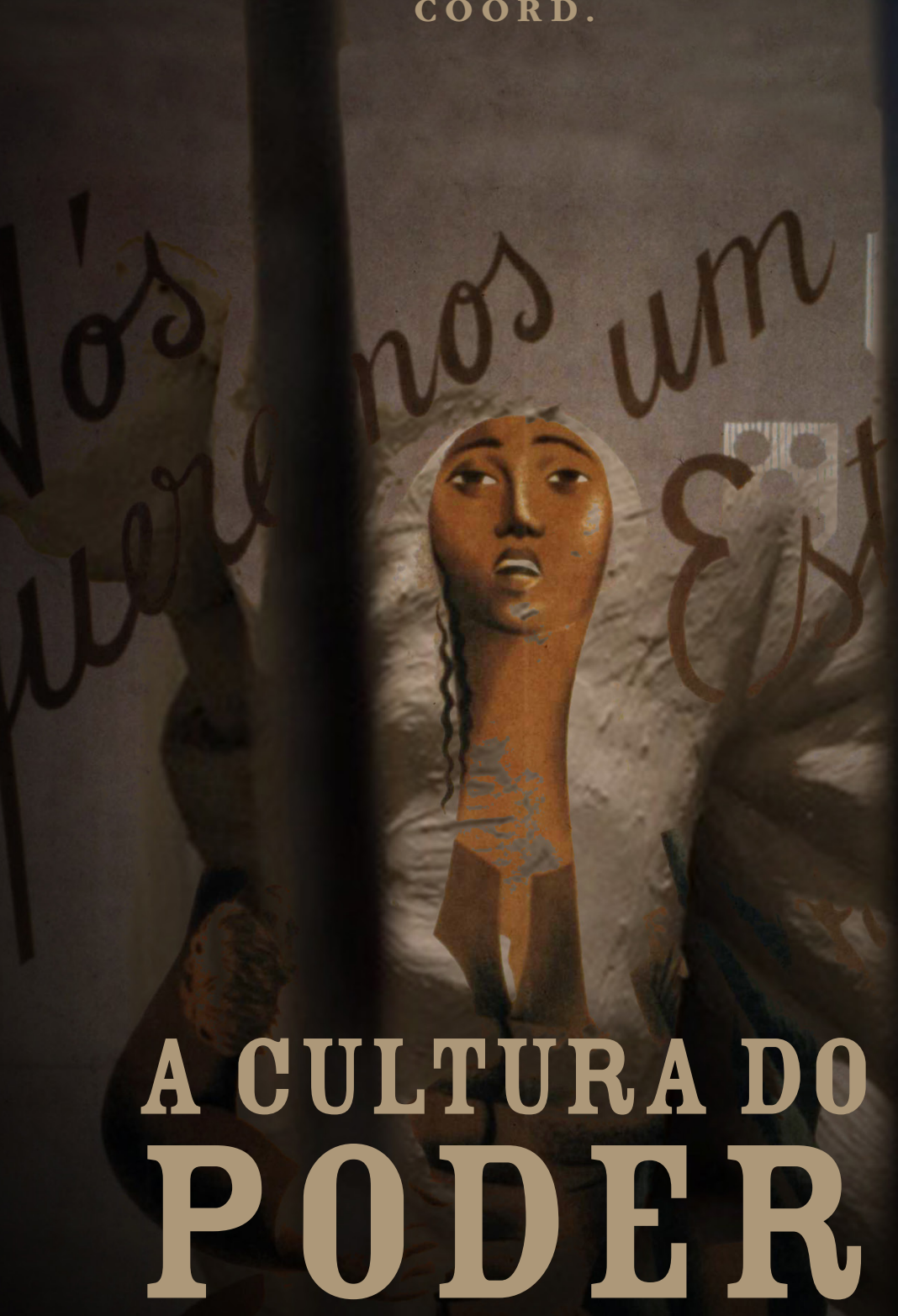

A PROPAGANDA INOS ESTADOS AUTORITÁRIOS 


\section{LA PROPAGANDA FASCISTA IN EUROPA. \\ A L C U N E R I F L E S S I O N I}

Alberto De Bernardi

\section{Propaganda e democratizzazione della società}

La storiografia è da tempo concorde sul fatto che la propaganda rappresenti un aspetto centrale delle dittature totalitarie del XX secolo, nella misura in cui le differenzia dai regimi autoritari che nell'età contemporanea si sono presentati sulla scena mondiale. Questa centralità vale in un duplice senso; da un lato lo studio della propaganda, sia sul versante delle forme e dei contenuti attraverso cui viene realizzata nei diversi regimi, sia su quello delle istituzioni e delle strutture che presidiano alla sua diffusionche consente di valutare quanto un regime possa essere collocato o meno nello spazio storico del totalitarismo; dall'altro permette di cogliere sia le omogeneità sia le specificità dei diversi totalitarismi facendo emergere con nettezza i miti ideologici sui quali si fonda non solo il consenso che li circonda, ma anche la loro autorappresentazione.

Per entrare compiutamente nel tema mi sembra che anche a distanza di più di cinquant'anni le riflessioni del sociologo statunitense Barrington Moore jr. sui fascismi rappresentino ancora una bussola utilissima. "Il fascismo - scrive nelle pagine conclusive della sua opera più famosa dedicata alle 'origini della dittatura e della democrazia' - è inconcepibile senza la democrazia, o meglio 
senza, come a volte viene retoricamente chiamato, l'ingresso delle masse sulla scena della storia. Il fascismo infatti è stato un tentativo di rendere popolari e plebei la reazione e il conservatorismo, il quale ultimo perse ovviamente attraverso il fascismo quel sostanziale rapporto che aveva con la libertà..."1 .

Moore si riferisce a quel processo di democratizzazione della società europea che ha cominciato il suo itinerario alla fine dell'Ottocento e che si è sviluppato nel quindicennio precedente la prima guerra mondiale, nella quale le classi lavoratrici entrano prepotentemente nello spazio pubblico sospinte non solo dalla progressiva affermazione del suffragio universale, ma anche dalla crescita dei consumi che le trasforma in consumatrici di beni industriali: oggetto quindi di una duplice propaganda, quella dei partiti e quella della pubblicità. In queste società si è verificata una trasformazione dei sistemi politici ottocenteschi, fondati su partiti elitari che organizzavano l'azione parlamentare di notabili rappresentanti più di interessi che di ideologie e per questo disponibili al trasformismo e al clientelismo. L'irrompere sulla scena di partiti di massa fortemente ideologizzati, come quello socialista, nazionalista o cattolico, cambia radicalmente il funzionamento del sistema rappresentativo e il ruolo dei parlamenti, come proprio in quegli anni aveva messo il luce Max Weber, ridimensionando $\mathrm{i}$ vecchi partiti notabilari, che non sono in grado nel nuovo contesto proprio perchè non dispongono delle risorse politiche indispensabili per operare nella società di massa: il professionismo politico e la militanza che struttura i nuovi partiti intorno a una rete capillare di circoli e sezioni, al tesseramento, a giornali e fogli di propaganda; le organizzazioni di secondo livello, come i sindacati e le altre molteplici forme di associazionismo di carattere ricreativo, sportivo,

\footnotetext{
${ }^{1}$ MOORE JR, B. Le origini sociali della dittatura e della democrazia. Proprietari e contadini nella formazione del mondo, Einaudi, Torino, 1969.
} 
femminili, che strutturano il tempo libero dei lavoratori, ma anche della piccola borghesia urbana e rurale, prima, ovviamente, che il cinema e la radio modificassero radicalmente la fruizione del tempo libero delle società industrializzate; l'ideologia che si propone come una nuova religione civile a cui è affidato il compito di definire i fini dell'agire politico collettivo e che costituisce, più che i programmi, il fulcro della propaganda.

La propaganda è la forma che assume la comunicazione politica nelle società di massa e che presuppone il partito politico che la organizza e la promuove per realizzare il suo obbiettivo fondamentale, rappresentato dalla mobilitazione politica dei gruppi sociali dei quali si tenta più che di esprimerne gli interessi, di plasmarne le aspirazioni e di riorientarne la tavola dei valori su cui si era basata l'integrazione tra popolo e stato nel XIX secolo. Come è ovvio, nel ventennio precedente la Grande guerra questi fenomeni non solo sono incipienti nell'intero continente, e si dispiegheranno compiutamente solo nel cinquantennio successivo, ma sono anche diversificati a seconda del grado di sviluppo economico degli stati europei, nella misura in cui la massificazione sociale è diretta conseguenza dell'industrializzazione.

In questo quadro i paesi mediterranei rappresentano il fanalino di coda dell'Europa - perché, salvo in parte l'Italia, sono ancora dominati da una economia prevalentemente agraria, ma non per questo non vengono permeati dai fenomeni di modernizzazione che il ciclo espansivo prebellico diffonde anche nelle periferie dell'Occidente. Anche qui, dunque, la partecipazione e la mobilitazione politica costituiscono il nuovo elemento centrale dei sistemi politici e contribuiscono a creare lo spazio pubblico nel quale si confrontano grandi forze politiche collettive animate da visioni del mondo, da ideologie, differenti. Cioè, detto in altri termini, gli individui, le persone sono in qualche modo proiettate nella politica in maniera stabile e "irreversibile". 
Il processo di democratizzazione della società comporta dunque un complesso fenomeno di politicizzazione di massa, che accentua la conflittualità sociale, perchè in gioco vi sono la difesa di antichi privilegi, tra cui quello che assegnava solo alle élites il diritto di "fare politica", cioè di contendersi la guida dello stato, insieme a quelli che garantivano una distribuzione del reddito radicalmente squilibrata a favore dei ceti proprietari, cui corrisponde un nuovo protagonismo delle classi subalterne, rafforzate strutturalmente dall'industrializzazione e dalla penetrazione del capitalismo nelle campagne.

Se in tutt'Europa il passaggio dal liberalismo alla democrazia, proprio per il processo di politicizzazione di massa che caratterizza questo itinerario, comportò una radicalizzazione dello scontro politico, in quella del Sud, anche per il carico di arretratezze che caratterizzava queste società quel passaggio raggiunse gradi di violenza e radicalità sconosciuti altrove: vengono assassinati due re, quello d'Italia nel 1900 e quello del Portogallo nel 1908, mentre quello di Spagna subisce nel '13 un attentato, mentre "Settimana tragica" di Barcellona del 1909 o la "settimana rossa" in Italia nella primavera del ' 14 con i loro elementi di carattere insurrezionale, ma anche con la scia di sangue che la repressione militare lasciò dietro di é confermano quanto la debolezza delle istituzioni liberali in questa parte dell'Europa impedisse di governare i conflitti sociali e politici dentro l'alveo di legittimità sancito dalle carte costituzionali particolarmente conservatrici, spingendoli ai margini dello spazio politico ancora in formazione, nei quali la lotta politica coincide con l'eversione e diventa problema di ordine pubblico' ${ }^{1}$.

Per queste ragioni in tutta l'Europa mediterranea il periodo che in Francia venne chiamato belle époque o negli Stati Uniti gilded

\footnotetext{
${ }^{1}$ Sulla crisi di fine secolo in Portogallo cfr. TORGAL, L.R. Estados novos, Estado novo. Coimbra: Imprensa da Universidade de Coimbra, 2009, t. II, pp. 15 e ssg.
} 
age, a segnalare un intreccio virtuoso di sviluppo economico, benessere, felicità pubblica e modernità, nei paesi che conosceranno poi l'avventura totalitaria, è stato invece un'epoca di crisi acuta, sia dei sistemi politici, sia delle relazioni tra gli individui e lo stato, determinata dalle contraddizioni che caratterizzano il processo di democratizzazione. Queste difficoltà però non devono offuscare il dato di fondo incontrovertibile che quel passaggio tra società elitari e società di massa avviene, anche nei paesi dell'Europa meridionale. E noi assistiamo alla formazione di sistemi politici, con la presenza organizzata di partiti e con una lotta ideologica tra democratici, conservatori, repubblicani, socialisti, radicali, liberali, nazionalisti; insomma, una articolata presenza di ideologie e di organizzazioni politiche.

In questo lo strumento di cui si servono queste organizzazioni politiche per veicolare la loro tavola di valori e la loro visione del mondo è la propaganda, che in quell'Europa di fine Ottocento inizio Novecento, sono essenzialmente i giornali, qui intesi in tutte le loro declinazioni - giornali politici, d'opinione, satirici, di intrattenimento, femminili, sportivi -: un complesso mosaico di fogli spesso artigianali, che escono con periodicità difformi e disomogenee, che nascono e muoiono continuamente, ma che in ogni caso garantisce una capillare diffusione dei programmi e dei progetti delle forze politiche direttamente proporzionale alla crescita della scolarizzazione anche tra i ceti popolari, volto al duplice scopo di creare opinione pubblica e mobilitazione politica. I giornali si affiancano alla "piazza", che costituisce la dimensione fisica dello spazio politico, luogo nel quale confluisce la protesta e nel quale i leaders incontrano le masse; un luogo dotato in se di una forza comunicativa per i suoi caratteri simbolici nel quale viene enfatizzata sia la creazione dell'opinione pubblica sia la mobilitazione politica. La piazza, associata alla barricata, era stata una invenzione della Rivoluzione francese, ma un secolo dopo assume un significato nuovo di campo di scontro dei 
partiti di massa e di luogo nel quale si struttura la comunicazione politica attorno ai capi dei movimenti politici organizzati. Mussolini o Hitler sono leader che si formano nella piazza e dalla capacità di controllarne le dinamiche derivano una quota considerevole della loro legittimazione politica; ma anche Lenin o Rosa Luxemburg sarebbero impensabili al di fuori di quello spazio nel quale si forma e si esercita la leadeship nelle società di massa e le idee politiche vengono trasformate in strumenti di consenso e di manipolazione delle folle. E questa dimensione spaziale della politica sarebbe stata centrale sino alla fine della società fordista negli anni ' 80 del secolo scorso.

\section{La nuova cultura della destra}

Ai fini del ragionamento che sto seguendo, e dunque di ricostruire il rapporto tra fascismo e propaganda, quel che qui mi preme sottolineare riguarda il fatto che nel nuovo contesto della società di massa nei due decenni che precedono la Grande guerra la novità più evidente è rappresentata dalla formazione di un pensiero e di un campo di forze di estrema destra progressivamente sempre più autonomo dal conservatorismo ottocentesco. Le idee forza introno a cui si struttura questa nuova rete di partiti e movimenti in Europa sono rappresentate da una nuova idea di nazione e una nuova idea di popolo rispetto a quelli che avevano dominato la scena politica nell'Ottocento.

Per seguire l'evoluzione della prima idea mi servo di una riflessione attribuita a Vilfredo Pareto, l'economista e sociologo italo-francese divenuto in quegli anni uno dei più famosi e significativi intellettuali della nuova destra europea e autore nel 1916 del celebre Trattato di sociologia generale. "A me pare - scriveva all'economista, suo amico e maestro Maffeo Pantaleoni - che i fatti dimostrano che il massimo errore del XIX secolo fu di credere che si potesse governare senza 
la forza; al quale errore capitale altri fanno corona: il suffragio universale, l'istruzione obbligatoria, il concetto di eguaglianza dei cittadini, ecc". Ciò che Pareto metteva in discussione era la connessione strettissima, che aveva accompagnato la creazione degli stati liberali, tra nazione e stato di diritto, tra nazione e libertà, tra nazione e umanità, che neanche i sussulti autoritari di fine secolo e di inizio Novecento avevano sostanzialmente messo in discussione. Il processo che emergeva nelle parole di Pareto consisteva nel fatto che in tutta Europa, l'idea mazziniana di nazione - cioè "l'universalità de' cittadini parlanti la stessa favella, associati, con eguaglianza di diritti politici, all'intento comune di sviluppare e perfezionare progressivamente le forze sociali e l'attività di quelle forze." - stava perdendo fascino e attrattiva per vaste aree dell'opinione pubblica.

In quell'epoca infatti lo spazio politico era ormai dal un decennio occupato da un flusso di opinioni e di teorizzazioni politiche, che metteva in discussione alla radice il "nazionalismo integrativo" che stette alla base dei risorgimenti nazionali europei. Questa fase si era conclusa con il 1870 quando, con la guerra franco-prussiana era emerso un nuovo tipo di nazionalismo, incarnato nel Reich tedesco, nel quale l'esaltazione della nazione si trasforma in una politica aggressiva rivolta a difenderne gli interessi a discapito delle altre nazioni e la patria comincia a coincidere con la razza, la forza, la potenza. Nei campi di battaglia intorno a Sedan, dove l'esercito francese venne sbaragliato da quello tedesco, la nazione cominciò a entrare in rotta di collisione con "l'umanità", vale a dire con quell'universalismo cosmopolita che aveva caratterizzato il patriottismo liberale e democratico fin dalla Rivoluzione francese.

In questa nuova ottica culturale che aumentava i suoi proseliti nelle classi dirigenti europee, la nazione, come scrisse proprio nel 1870 il grande antichista Fustel de Coulange non era più "ciò che si

${ }^{2}$ L'espressione è dello storico Louis Snyder The meaning of nationalism, 1954. 
ama”, cioè un ideale liberamente scelto, ma una terra e una razza, una identità collettiva che si è venuta plasmando attorno a delle tradizioni storiche, a un ambiente naturale, a una lingua e nel quale ciascuno si trova naturalmente collocato. Nel passaggio dal patriottismo umanitario ottocentesco a questo nuovo nazionalismo la nazione diventava un'ideologia che insieme ad altri elementi, contribuiva alla definizione della nuova tavola dei valori degli stati impegnati nella corsa imperialista e nella costruzione dell'economia-mondo protezionista.

Alla convinzione che nel futuro del mondo ci fosse la pace e che l'umanità, pur divisa in tante comunità nazionali, fosse destinata a convivere nel rispetto reciproco fondato sul comune riconoscimento dell'uguaglianza tra gli uomini, si andava progressivamente sostituendo una visione del mondo nella quale gli stati nazionali erano coinvolti in una lotta spietata per la supremazia, nella quale solo pochi erano destinati a emergere e tutti gli altri a soccombere. In questo contesto conflittuale la guerra veniva esaltata come lo strumento per eccellenza per risolvere i conflitti. Ma già a Sedan era emerso con forza che l'esperienza bellica poteva diventare lo strumento attraverso il quale cementare l'identità nazionale, e con il quale il "corpo mistico" della nazione poteva essere forgiato e introiettato nella mentalità collettiva. Un cambiamento di scenario geopolitico e di equilibri economici si era dunque tradotto in un mutamento di prospettive culturali e di visioni del mondo, che avrebbero dominato lo spazio pubblico continentale fino alla conclusione della Seconda guerra mondiale, e di cui in Italia l'intera esperienza crispina era stata fortemente impregnata.

Il percorso che andò separando l'idea di nazione dalla "religione della libertà" del liberalismo, fu l'esito di una serie di processi strutturali e politici, che riguardò sul piano culturale l'azione di una nuova generazione di intellettuali, nata grosso modo intorno agli anni ' 70 dell'Ottocento, attiva in molti paesi europei. Già alle 
soglie del nuovo secolo essa aveva rifiutato quella comune matrice di stampo positivista, sulla quale si era fondato il dialogo tra liberalismo progressista e socialismo, non solo in Italia ma in tutta Europa, nella seconda metà del XIX secolo. Per questa generazione, affascinata più da Bergson e da Nietzsche che da Marx e da Hegel, l'irrazionalismo vitalista e soggettivista che la viene plasmando culturalmente si tradusse in una frattura con la democrazia, cui faticosamente l'Europa liberale stava approdando: democrazia non tanto mazziniana, quanto piuttosto nella sua nuova accezione di democrazia di massa, inclusiva e antielitaria, aperta all'azione dei partiti, plasmata dall'accesso ai consumi, quale si stava delineando in tutto l'Occidente. Il rifiuto degli esiti democratici, cui il liberalismo, sotto la spinta della mobilitazione sociale delle classi lavoratrici soprattutto, operaie, stava approdando, si tradusse in un presa di distanza radicale da tutto ciò che sul piano delle istituzioni politiche e della tavola di valori aveva rappresentato questo complesso itinerario storico: il parlamentarismo, il liberismo economico, la pace, l'associazionismo sociale, lo stato di diritto ${ }^{3}$.

La trasformazione dei valori e delle rappresentazioni collettive per quel che concerneva l'idea di nazione fu sollecitata in modo determinante dall'imperialismo e dal protezionismo. Alla visione ideale di un pianeta integrato in un mercato aperto (presupposto di una dialettica pacifica tra le nazioni) si sostituiva la realtà di un mondo frammentato e diviso tra imperi mondiali, protetti da dazi e gabelle, in lotta fra di loro per il controllo delle materie prime e per i mercati di smercio delle produzioni industriali. Ne fu colpito a morte il cosmopolitismo progressista che era stato quasi un articolo di fede tanto per il pensiero democratico, quanto per quello liberale, e in larga misura anche per quello socialista ${ }^{4}$.

3 Sull'eredità di questo pensiero nella ideologia politica di Mussolini cfr. GENTILE, E. Le origini dell'ideologia fascista. Bologna: Il Mulino, 1996, pp. 61-110.

${ }^{4}$ Sul tema cfr. anche STRENHELL, Z. Né destra, né sinistra, Milano, Baldini e Castoldi, 1997. 
Cominciava così a prendere forma nella coscienza di gruppi di giovani intellettuali l'ideologia dello "Stato forte", all'interno contro il socialismo e contro le spinte emancipazioniste del lavoro, all'esterno contro le altre nazioni e le altre razze. "La vita - scrisse Nietzsche in Al di là del bene e del male (1885) - è essenzialmente appropriazione, offesa, espropriazione di tutto quanto è più debole, oppressione, durezza ...; stabilire un'uguaglianza tra la propria volontà e quella dell'altro ... può diventare una buona costumanza tra individui ... Ma appena questo principio volesse guadagnare terreno...come principio basilare della società, si mostrerebbe immediatamente per quello che è: una volontà di negazione della vita, un principio di dissoluzione e di decadenza" 5 .

Nel caso italiano alle spalle di questa nuova cultura politica che si stava facendo strada tra le élites intellettuali italiane si può rintracciare anche la teoria delle élites elaborata dal filosofo della politica Gaetano Mosca. Nel 1896 erano infatti usciti gli Elementi di scienza politica, dove il giurista palermitano espose in maniera compiuta la sua teoria delle élites, che rappresentava la critica più radicale della democrazia, intesa come espressione massima della sovranità popolare. Mosca riteneva impossibile nei fatti il metodo democratico, perché rintracciava nella storia una legge universale secondo la quale il governo reale era sempre stato saldamente nelle mani di una minoranza organizzata, la cosiddetta "classe politica", che era riuscita, al di là dei sistemi elettorali vigenti, a controllare e a guidare la maggioranza del popolo.

Per ottenere questo risultato erano indispensabili due condizioni: la prima riguardava la necessità di giustificare il potere politico alla luce di un progetto, di una missione, di una ideologia, potremmo dire oggi, senza la quale la massa disarticolata dei governati non

\footnotetext{
5 Su queste tematiche mi permetto di rimandare a DE BERNARDI, A. GANAPINI, L. Storia dell'Italia unita. Milano: Garzanti, 2010, pp. 965-970.
} 
poteva essere mobilitata; la seconda consisteva nella capacità della classe politica di aggregare attorno a se il consenso di un ceto medio abbastanza diffuso, dotato di un certo benessere economico e di una livello culturale adeguato. Interprete più coerente di questo esito rivoluzionario e antiliberale del pensiero di Mosca fu proprio Vilfredo Pareto, che in quel Trattato di sociologia generale che abbiamo gia citato elevò una sorta di panegirico all'azione politica delle aristocrazie, che assumono la direzione politica di un paese e la mantengono fino a quando non verranno scalzate da altre élites: la storia in quest'ottica si riduceva a questa lotta incessante di aristocrazie forti e aggressive, che non ha nessun fine "progressivo", al di là delle giustificazioni che esse di volta in volta elaborano, perché la vicenda umana è dominata da azioni irrazionali e da istinti collettivi. Quando Pareto scrisse il suo libro il parlamentarismo italiano era ormai entrato in una crisi che di i a pochi anni si sarebbe rivelata irreversibile e "l'appello al popolo" di stampo bonapartista, che di fatto ne rappresentava l'alternativa, si era già imposto nei fatti come pratica politica reale, spingendo l'Italia in guerra e sancendo il trionfo "extraparlamentare" dell'interventismo.

$\mathrm{Ma}$, come accennato in precedenza, questa nuova ideologia politica della destra nazionalista e poi fascista è accompagnata da una altra trasformazione culturale profonda che riguarda l'idea di popolo. Questo termine non indica più l'insieme di cittadini consapevoli e liberi, il cui allargamento all'intera universalità degli abitanti di una nazione era il compito principale dei governi, ma una folla indistinta e mobile, passionale e disgregata, tenuta insieme dall'ordine della produzione, dagli stimoli standardizzati dei consumi e dalla forza del potere politico. L'indagine sulla folla divenne il terreno scientifico e culturale su cui si vennero ridefinendo le scienze sociali, a partire dal lavoro fondamentale del sociologo francese Gustave Le Bon, la Psychologie des foules del 1895, che inaugurava una delle più complesse concettualizzazione che furono alla base 
di tutti i fenomeni totalitari: la folla è una nuova potenza sociale, instabile e irrazionale, priva di senso di responsabilità e di freni inibitori, del tutto inadatta a essere governata dalle strumentazioni istituzionali inventate dal liberalismo. L'Italia già qualche anno prima della pubblicazione dell'opera di Le Bon era diventata il laboratorio della teoria delle folle con i lavori pionieristici di Enrico Ferri, Scipio Sighele e Guglielmo Ferrero dedicati alle folla "delinquente" e alla psicologia collettiva. Soprattutto questi ultimi due elaborano immediatamente le implicazioni politiche di questa nuova teoria. In due pamphlet dal titolo inequivocabile, Contro il parlamentarismo e La reazione, uscite entrambe nel 1895, il cui asse concettuale era l'estraneità della folla alla libertà e l'attrazione per un "genio" cesarista, capace di guidarle perché era in grado di coglierne gli istinti e i sentimenti. La folla era dunque una "femmina" cieca che andava plasmata dall'altro dall'azione di minoranze e di capi risoluti che possedevano i nuovi strumenti culturali per pensare alla politica nell'età della massificazione sociale: forza, miti, autorità ${ }^{6}$.

Questi strumenti dovevano però fare capo ad una nuova forma di Stato, che fosse portatrice di quei valori unificanti e di quei fini collettivi che la società della folla non era più in grado di esprimere autonomamente: uno stato "organico", per dirla con il filosofo Giovanni Gentile, il più "organico" al fascismo tra gli intellettuali italiani, capace di unificare la nazione attorno a uno destino comune di cui era l'unico depositario e di cui gli individui diventavano artefici" riconoscendosi nel suo primato.

La prova che queste teorizzazioni coglievano lo spirito dei tempi più di ogni altra cultura politica emerge dalla fortuna che ebbe tra le fila del socialismo italiano ed europeo il pensiero di George

\footnotetext{
${ }^{6}$ Sulla critica al parlamentarismo in Europa nei due decenni a cavallo della Prima guerra mondiale cfr. MAZOWER, M. Le ombre in Europa. Democrazie e totalitarismi nel $X X$ secolo. Milano: Garzanti, pp. 30 e ssg.
} 
Sorel, figura di spicco del "revisionismo" marxista inaugurato in Germania da Eduard Bernstein. Alla fine del secolo sotto la spinta di una progressiva adesione alla svolta antipositivistica della cultura europea, elaborò una teoria della rivoluzione proletaria interamente incentrata sull'azione volontaristica, sullo "slancio vitale" delle masse e sulla violenza proletaria, come strumento fondamentale per la distruzione della società borghese: la rivoluzione non percorreva la storia come una "talpa", secondo una definizione di Marx, pronta a emergere, quando condizioni storiche oggettive l'avrebbero resa possibile, ma esplodeva quando la classe operaia era in grado di trasformare la sua forza creatrice in un possente atto rivoluzionario. Ed erano i miti, propagandati con efficacia tra le masse, il vero vettore della rivoluzione, più che le contraddizioni dello sviluppo economico. Intorno a Sorel e al suo progetto anarcosindacalista si strinsero componenti crescenti del movimento operaio italiano, antiriformiste e antimarxiste, tra le cui fila si affermò la personalità politica di Benito Mussolini, prima capo dell'ala rivoluzionaria del partito e poi leader dell'interventismo nazionalista.

\section{La guerra mondiale come mobilitazione di massa}

Ovviamente la guerra mondiale è un grande acceleratore di democratizzazione e di mobilitazione politica, sia perché mette in movimento oltre 60 milioni di giovani tra europei e abitanti delle colonie, sia perché per la prima volta la guerra, per i suoi caratteri del tutto nuovi, richiede una legittimazione di massa: bisogna infatti convincere i soldati che è giusto combattere, che sono giusti i valori per i quali si muore, promuovendo tra i soldati un complesso processo di identificazione con la ragioni della guerra. La guerra cioè deve apparire come "giusta" cioè rimandare alla difesa o all'affermazione di una tavola di valori che ruota intorno a quella nuova idea di nazione di cui 
abbiamo parlato poc'anzi. Per i tedeschi, come ha insegnato Thomas Mann nelle pagine memorabili di Considerazioni di un impolitico, la guerra era giusta perché prosegue uno scontro secolare tra Kultur e Civilisation, iniziato nel 1798 e che ha per oggetto l'estraneità dello spirito germanico alla democrazia; per la Francia era l'esatto opposto: l'union sacréé del popolo stretto intorno proprio ai valori della repubblica democratica e progressista.

Per l'Italia il conflitto era la "quarta guerra del Risorgimento" per portare a compimento l'unificazione nazionale, ma anche l'occasione di ribadire la propria missione di grande potenza europea e mediterranea iniziata con la guerra coloniale di Libia del 1911, cantata dal poeta Giovanni Pascoli in una poesia che veniva imparata a memoria in tutte le scuole del Regno dal titolo inequivocabile La grande proletaria si è mossa. Come è noto l'interventismo italiano era diviso in due fronti: quello democratico che voleva la guerra per lottare contro le grandi potenze assolutistiche e riaffermare il rapporto tra il Risorgimento e la democrazia; quello nazionalista che mitizzava la guerra come "lavacro" nel quale rigenerare la nazione liberandola dalle scorie del liberalismo imbelle e borghese e renderla disponibile a riconoscersi nel nuovo destino di potenza europea. Rapidamente si impose questo secondo orientamento e l'ideologia della "grande guerra" divenne il filo della propaganda che centinaia di giornali diffusero tra i soldati nelle trincee ${ }^{7}$.

I "giornali di trincea", di cui si è occupato Mario Isnenghi molti anni fa con un libro destinato a cambiare l'approccio storiografico al primo conflitto mondiale ${ }^{8}$, costituiscono l'osservatorio privilegiato del ruolo della propaganda nel processo di formazione del soldato

${ }^{7}$ Sul rapporto tra guerra e nascita della destra portoghese cfr. PAIS DE SOUSA, J., o fascismo catedratico de Salazar. Coimbra: Imprensa da Universidade de Coimbra, 2011, pp. 124-160. Sul caso Italiano vedi VENTRONE, A. La seduzione totalitaria. Roma: Donzelli, 2003.

8 ISNENGHI, M. Giornali di trincea. 1915-1918, Torino, Einaudi, 1977. 
non solo come combattente, ma come cittadino delle nuove nazioni plasmate dal conflitto. Un enorme sforzo di manipolazione della coscienza collettiva nella quale l'evento-guerra viene rielaborato non solo per rendere accettabile il massacro di massa che stava falcidiando un'intera generazione di giovani europei ${ }^{9}$, ma soprattutto trasformare la guerra in un mito politico su cui plasmare la nazionalizzazione delle masse. Non si trattava dunque soltanto di "normalizzare l'abnorme e il mostruoso" di questa spaventosa strage, per motivare i soldati al combattimento ripescando nelle pieghe della mentalità collettiva tutti gli stereotipi militareschi o patriottici per valorizzare la guerra e distruggere l'immagine del nemico, ma di "culturalizzare grandi masse di uomini strappandoli alla loro cultura precedente" proiettandoli in una nuova cultura di massa fatta di "nichilismo e di misticismo, di risolutezza e di sradicamento, di credulità e cinismo che sarà la comune essenza del tipo del fascista, del bolscevico, del nazionalsocialista", che disintegrerà la vecchia cultura liberale e socialriformista ${ }^{10}$.

È oramai opinione comune tra gli storici che la grande guerra abbia rappresentato l'evento nel quale si concentra al più alto livello la modernità novecentesca, per l'intreccio di massificazione, industrializzazione e stato nazionale, e che la propaganda di guerra cerca di rendere senso comune. Le due ideologie che la guerra spigiona, il fascismo e il bolscevismo, sono dunque figlie di questa modernità. Per molti aspetti esprimono dunque i cambiamenti che sul piano sia socio-economico, che simbolico quel evento traumatico aveva prodotto nella coscienza europea apparendo più "attuali" delle altre culture politiche precedenti, che erano invece eredità dell'Ottocento. Bolscevismo e fascismo sono dunque prodotti della

\footnotetext{
${ }^{9}$ Sul tema cfr il fondamentale, LEED, E. J., Terra di nessuno. Esperienza di guerra e identità personale nella prima guerra mondiale. Bologna: il Mulino, 1985.

10 GALLI DELLA LOGGIA, E. Introduzione all'edizione italiana, in: Fussell, La grande guerra e la memoria collettiva. Bologna: il Mulino, 1984, p. XVII.
} 
guerra mondiale anche perché in quella modernità essa ha inserito alcune componenti fondamentali che avranno un ruolo decisivo nel plasmare le ideologie di quei due movimenti: la violenza come strumento di lotta politica, demonizzazione del nemico come leva per costruire l'identità del corpo dei militanti e la propaganda come manipolazione della folla al fine di cementarne l'adesione fideistica alla religione civile professata dai movimenti.

Come è noto, è Mussolini a inventare del termine totalitario, che per molti aspetti deriva dai caratteri "totali" assunti dalla Grande guerra, che ne avevano fatto un evento bellico assolutamente incomparabile con quelli precedenti, nella misura in cui quel termine evocava l'identificazione assoluta tra stato e il popolo che si era realizzata nelle trincee e che ora il fascismo voleva riprodurre come valore politico fondamentale del rapporto tra nazione e fascismo.

Il tema dell'identificazione tra regimi totalitari e masse non riguarda ovviamente solo il fascismo; lo troviamo fin dalle origini di ogni esperienza politica che si rifà a questo modello idealtipico, sia comunista che fascista, e caratterizza molte esperienze politiche che di quei regimi furono gli embrioni: dall'Action Française, a Primo de Rivera, dal nazionalismo radicale italiano ai gruppi di estrema destra tedeschi da cui uscì il nazionalsocialismo hitleriano.

\section{La religione civile dei fascismi}

Ma anche la religione civile dei fascismi è una visione del mondo totalizzante, nella misura in cui si incarica di descrivere, interpretare, riassumere tutti i fenomeni della vita individuale e collettiva e di iscriverli in una interpretazione della storia che ponga quei regimi come esito del lungo cammino delle nazioni e come depositari dei 
fini ultimi che sono assegnati ai singoli popoli-razza, siano essi lusitani o italici, ariani o ispanici ${ }^{11}$.

Questo contesto ideologico del tutto nuovo enfatizza ulteriormente il ruolo della propaganda politica perché è il veicolo attraverso cui ogni regime si propone di attivare quella identificazione tra il popolo e il destino comune di cui i fascismi sono interpreti, che costituisce il cuore del suo progetto politico. E questa enfatizzazione è notevolmente incrementata dalle nuové tecnologiche che la modernità mette a disposizione del potere politico: un ventaglio di strumenti nuovi, dal cinema alla radio e successivamente la televisione, combinati ad un uso altrettanto nuovo di quelli più tradizionali, come la fotografia, le arti visive e gli stessi giornali, tutti trasformati i fogli del partito-stato. Questa vasta gamma di strumenti propagandistici chiama in causa direttamente il ruolo di una nuova leva di intellettuali di regime che diventano esperti di comunicazione di massa assai diversi da quelli del passato: registi, sceneggiatori, attori, giornalisti, ideatori e conduttori di trasmissioni radiofoniche, pubblicitari, che si affiancano a pittori, architetti e scrittori in uno sforzo di comunicazione politica totalizzante ${ }^{12}$.

La propaganda è uno dei due "occhi" con cui il regime esercita il suo controllo sociale: l'altro è la polizia politica e segreta, che controlla i comportamenti collettivi; la propaganda cerca piuttosto di crearli, di costruire 1' "uomo nuovo" fascista. Propaganda e repressione sono dunque due facce della stessa medaglia: la prima crea e diffonde la religione civile, la seconda colpisce il dissenso e crea quel clima di paura e di assenza di alternative nel quale quella religione si può rapidamente diffondere.

11 Su questo mi permetto di rimandare al mio, Una rivoluzione moderna. Il fascismo come problema storico. Milano: Bruno Mondadori, 2006.

12 Sul rapporto tra intellettuali e fascismo, cfr. ISNEGHI, M. Intelletuali milianti e intellettuali funzionari. Appunti sulla cultura fascista. Torino: Einaudi, 1979 e TORGAL, Op. cit., t. II, pp. 71-116. 
È bene però a questo punto interrogarsi su quali siano i punti salienti di questa religione civile fascista nella convinzione che essa abbia una tavola di valori comune che sia possibile rintracciare in tutte le diverse esperienze storiche nella quale essa si e concretizzata.

Innanzitutto al centro vi è il tentativo di sostituire alla classe la nazione, come baricentro della vita collettiva in diretta competizione con il comunismo, al quale si intende inoltre togliere il monopolio della rivoluzione. I fascismi si presentano dunque come movimenti rivoluzionari, portatori di una trasformazione altrettanto radicale della società che mette in campo la stessa carica distruttiva nei confronti del passato borghese e liberaldemocratico, in nome però di una riscoperta della tradizione.

A questo proposito basti pensare alla idealizzazione della ruralità e del mondo contadino, depositari di valori di frugalità, familismo, tradizionalismo, obbedienza, che Mussolini, quanto Salazar o Franco mettono al centro del loro messaggio ideologico, contrapponendoli a quelli urbani e industriali, mentre fanno delle campagne il luogo nel quale si sperimentano nuovi progetti di modernizzazione agricola. Se per Lenin lo slogan che compendiava il bolscevismo era "i soviet più l'elettrificazione", cioè il rapporto tra il nuovo potere proletario e lo slancio modernizzatore dell'industria, per i fascismi se ne potrebbe creare un altro incentrato sul nesso tra famiglia contadina e corporativismo; corporativismo qui inteso più che come modello economico, come alveo ideologico nel quale sussumere e annullare il conflitto sociale che costituiva l'elemento costitutivo del paradigma classista ${ }^{13}$.

Certamente dietro il mito della ruralità vi è quello della razza, nella misura in cui il contadino-produttore rappresenta il modello dell' "uomo nuovo" fascista: un homo faber che è anche il prototipo del

$13 \mathrm{U}$ corporativismo nell'ideologia fascista cfr oltre al testo gia citato di L. R. TORGAL, ZUNINO, P. G. Ideologia del fascismo. Miti, credenze e lavori, della stabilitazione del regime, Bologna: Il Mulino, 1985. 
soldato ereditato dalla guerra mondiale, combattuta essenzialmente da giovani contadini, sintetizzato nella mistica fascista italiana dallo slogan scritto su tutti i muri delle case rurali, "è l'aratro che traccia il solco, ma è la spada che lo difende". Il contadino-soldato è il soggetto rivoluzionario della rivoluzione fascista come l'operaio di fabbrica lo è per quella bolscevica, perché incarna il mito della terra e del sangue che sta alla base del razzismo fascista e potremmo dire antropologicamente estraneo al mito internazionalista del proletariato senza nazione proprio del marxismo. Quello comunista è l'ultimo erede della cultura della rivoluzione francese, cosmopolita, umanitaristica, progressista quella fascista è figlia della cultura del "1914", totalitaria, organicista, reazionaria.

Il contadino-soldato rimanda a un altro mito fondamentale dei fascismo: l'impero inteso nel duplice senso di politica di potenza e di mito fondativo dell'identità nazionale. Questo mito, che è al centro della propaganda fascista, si muove di diversi livelli. Nel caso portoghese è idea-forza nella quale si riassume soprattutto nel secondo dopoguerra la difesa di una sorta di "stato di eccezione" del Portogallo rispetto al resto del continente e a cui è affidato il compito di giustificare il salazarsimo come dittatura "sopravvissuta" in una Europa che si integra e nella quale non c'è piu spazio per il fascismo. In Spagna è una nostalgia ideologica di un "passato che non vuole passare" e che il regime alimenta per proporsi come erede di una tradizione di grande potenza mondiale, irrecuperabile e irriproponibile. Nel caso italiano è l'obbiettivo centrale della politica estera fascista che guida la ripresa del colonialismo con la conquista dell'Etiopia nel 1936 e soprattutto l'intervento nella seconda guerra mondiale, che aveva per obbiettivo la trasformazione del Mediterraneo in un area di influenza italiana. Sia il fascismo italiano che il salazarismo crolleranno per difendere il mito imperiale, ma questo non può fare velo sul fatto che esso abbia rappresentato uno 
snodo centrale della retorica politica di questi regimi e un punto di forza del consenso che si sviluppa intorno ad essi.

Infatti l'impero era una costruzione politico-culturale complessa, che non solo evocava lo "spazio vitale" che ogni nazione fascista riteneva di dover conquistare o difendere per garantirsi il futuro di grande potenza. Al di à dei miti del passato evocati dalla propaganda, l'impero era in realtà un'idea politica moderna perchè presupponeva l'esistenza di una nazione e di una ideologia, cioè di due concettualizzazioni proprie della modernità, capaci di imporre una propria visione del mondo e una propria egemonia politico-militare ad altri stati più deboli e privi di forze propulsive interne, ad essa circostanti. Al centro del Grossraumordnung (grande spazio), per dirla con Carl Schmitt, stava lo Stato-Nazione totalitario, in grado di imporre ad una area territoriale, la cui vastità dipendeva solo dalla capacità, tecnica, economica e organizzativa di quest'ultimo di dilatare la propria potenza espansiva, il proprio controllo e il proprio dominio. Si creava così una gerarchia tra le nazioni, dove poche appartenevano al rango di stati imperiali e dove molte erano ridotte a "vassalli", privi nei fatti di sovranità: ma una nazione senza sovranità di fatto perde questo status, per ridursi a un mero contenitore territoriale, privata com'è di definire la propria politica estera, di riconoscersi in una autonoma tavola di valori, di definire un proprio modello di sviluppo e una propria organizzazione sociale. Gli imperi si configuravano come gigantesche sfere di influenza di poche grandi nazioni, unici effettivi soggetti del diritto internazionale e della sovranità, e al contempo come spazio in cui un popolo "spazialmente" e politicamente consapevole poteva esercitare la sua egemonia

Era questi il mito politico perseguito da Salazar, da Mussolini o da Hitler, declinato dentro le diverse storie nazionali e nei diversi contesti internazionali.

Basti pensare alla esaltazione dell'impero romano nella mitologia politica del fascismo, proposta come eredità e al contempo come 
esito del fascismo: un ritorno al passato che era anche un progetto di ricostruzione su cui misurare la grandezza del regime. Inserire il culto di Roma, come mito politico del fascismo e perno della sua "religione" politica in quel continuum tra la storia italiana e la romanità, che aveva rappresentato un altro "filo rosso" della storia insegnata. Il "ritorno all'Africa" di Mussolini "con la stessa anima di Roma antica", divenne un leitmotiv dell'ultimo nuovo capitolo aggiunto in tutti manuali scolastici dopo il 1936, che diventavano così uno dei più potenti strumenti di propaganda politica. In esso emergevano sia l'esaltazione della politica di potenza del fascismo erede dei fasti imperiali di Roma, sia il richiamo alla guerra perenne tra le nazioni nella quale si era finalmente impegnata anche l'Italia, sia, infine, la retorica della missione civilizzatrice, secondo la quale come scriveva Pietro Silva, uno degli storici di regime più accreditati, nel suo Corso di storia per i licei - "le popolazioni delle regioni occupate accoglievano gli italiani come liberatori".

Con questo bagaglio di certezze e di aspettative i manuali di storia accompagnarono le giovani generazioni all'appuntamento con la guerra mondiale, nel corso della quale gran parte di loro avrebbe dovuto tragicamente constatare non solo e non tanto il servilismo e la pochezza morale, prima ancora che scientifica, di questa generazione di storici fascisti, quanto la debolezza del consenso di cui il fascismo ancora godeva. Ancora nel ' 41 la conclusione del manuale del Manaresi, quando già erano evidenti i segni dell'imminente crollo del regime sotto le macerie di una guerra perduta, testimonana quanto ancora quel mito fosse nella propaganda del regime.

Nell'Europa odierna, travagliata dagli odi politici e dalle contese sociali, l'Italia stretta al suo duce, appare oggi come un oasi di ordine e grandezza. Essa è oggi il simbolo dell'avvenire politico del mondo intero, poiché il fascismo ha creato una nuova forma di stato autoritario e corporativo, che si sostituirà allo stato 
liberale e democratico, portato della rivoluzione francese, ma ormai superato dalle nuove esigenze della moderna vita politica e sociale.

L'impero è dunque un potente strumento ideologico per fascistizzare la società, sia come sforzo per indottrinare i cittadini sia come tentativo di far credere che quei miti fossero non mere costruzioni ideologiche, ossessivamente messe in scena in quel palcoscenico di cartapesta rappresentato dallo spazio pubblico totalitario, ma propositi effettivamente raggiungibili e coerenti per uno stato-nazione presentato come una grande potenza inespressa.

Il fascismo italiano si avvalse per quell'opera innanzitutto del partito, a cui venne affidato un compito di incessante pedagogia collettiva, attraverso la rete capillare dei grandi organismi di partecipazione passiva creati dal regime, volti a mitizzare $i$ fasti imperiali del regime: una macchina colossale costituita da un pletora di organismi di massa che irregimentavano i cittadini "dalla culla alla bara". A fianco del partito e spesso in competizione con esso opera il Minculpop, il Ministero della cultura popolare, istituito nel 1937, che controllava le notizie e il modo di comunicarle attraverso tutti i giornali, nazionali e locali, potendo contare su un esercito di giornalisti "militanti" o succubi, disposti quotidianamente a trascrivere le veline (direttive diffuse in più copie su carta velina) che provenivano dal centro. Lo stesso valse per i programmi radiofonici e per i film che venivano immessi del circuito delle sale cinematografiche. Con la creazione, nel 1937, di Cinecittà, la città del cinema alle porte di Roma, le istanze propagandistiche si combinarono con la creazione di una moderna industria cinematografica, in grado di realizzare film di ottima qualità, nei quali emerse una nuova generazione di artisti, che avrebbe animato la produzione cinematografica del dopoguerra. A Cinecittà il regime si impegnò a promuovere il cinema nazionale, 
dedicandosi soprattutto alla ricerca di capitali, indispensabili per sostenere le grandi operazioni propagandistiche del regime sfociate in Scipione l'Africano, un colossal incaricato di proiettare l'impero fascista nell'immaginario collettivo. Nel 1937 a Cinecittà furono prodotti diciannove film; nel 1940 divennero quarantotto e ancora nel 1942 dagli studi romani uscirono ben cinquantanove film, a dimostrazione di quanto elevato fosse l'investimento sulla propaganda cinematografica anche quando ormai il regime era agli sgoccioli.

\section{La propaganda tra partito e stato}

Ovviamente tutti questi elementi della religione civile si declinano in maniera diversa nelle concrete esperienze storiche dei fascismi, le cui specificità sono legate sia al diverso grado di sviluppo raggiunto, cioè al diverso grado di modernità che connotava i singoli regimi, sia alle forme storiche con cui essi si sono affermati nei singoli contesti nazionali, da cui discesero i diversi quadri istituzionali che li caratterizzarono, sia infine dai diversi profili ideologici che essi assunsero. Ovviamente queste puntualizzazioni rimandano al fatto che anche i regimi fascisti, come quelli democratici o comunisti, sono imperfetti, nel senso che non realizzano i fini che sono costitutivi delle religioni civili che li incarnano e quindi sono sempre inadeguati rispetto ai modelli politologici elaborati dalle scienze sociali per interpretarli.

A proposito della propaganda, emerge subito una differenza tra i regimi fascisti italiano e tedesco, e i regimi fascisti iberici, che riguarda il ruolo del partito come organizzatore della comunicazione politica, forte nel caso fascismo italiano, più debole nei fascismi iberici definiti appunto regimi totalitari senza partito. Ma questa differenza non deve però ingenerare dei fraintendimenti perchè, in tutti questi regimi, è lo stato il vero soggetto che si assume 
l'onere della propaganda politica utilizzando una complessa gamma di strumenti, tra cui, dove c'è, il partito unico. La storia della radio e della televisione mette bene in evidenza questa centralità dello stato totalitario come erogatore diretto di propaganda perché in questi caso vengono costituiti enti appositi che forniscono i servizi radiofonici e televisivi, nei quali finalità politiche, intrattenimento e formazione culturale si intrecciano portando a livelli prima sconosciuti la manipolazione della coscienza collettiva, che rimarrà una elemento costitutivo delle comunicazioni di massa anche quando il totalitarismo sarà scomparso dalla scena politica. 